\title{
High-Order Consensus Seeking: A Frequency Domain Approach
}

\author{
Bo Yang* \\ School of Automation, Wuhan University of Technology, Wuhan 430070, China
}

Received: 9 Aug. 2013, Revised: 11 Nov. 2013, Accepted: 12 Nov. 2013

Published online: 1 Jul. 2014

\begin{abstract}
High-order consensus, in which individual high-order dynamic units keep in pace with each other in a distributed fashion, depends both on the feedback gains of the protocol and on the properties of the interaction network. By employing a frequency domain method, we explicitly derive analytical equations that clarify a rigorous connection between the stability of general high-order consensus and the system parameters such as the network topology and feedback gains. Using the derived consensus polynomials, the general sufficient and necessary stability criterion is obtained for high-order consensus networks of arbitrary topology. Furthermore, a sufficient condition of desirable time complexity for high-order consensus is given by exploiting the topology properties of underlying networks. Numerical simulation results are provided to demonstrate the effectiveness of our theoretical results.
\end{abstract}

Keywords: high-order consensus, frequency domain, distributed cooperative control, stability criterion

\section{Introduction}

Recent years have witnessed vibrant advances in our understanding of distributed cooperative control for dynamic networks $[1,2,3,4,5,6]$. For coordination strategies to be successful, many issues must be addressed. Among these different issues, the research on shared information of interest in the network of dynamic agents facilitates significantly the distributed coordination $[4,7,8,9,10,11,12]$. Therefore, an important issue for coordination is to design effective protocols so that the dynamic agents in the network are able to converge to a consistent sense or view of the shared information of interest in the presence of limited information exchange and communication time-delays. Although originally studied in the field of computer science (particularly in distributed computation and automata), the consensus problems discussed above have been studied extensively in the context of distributed coordination of networks of dynamic agents partly due to the potential broad applications in various areas including congestion control in communication networks, cooperative control of multiple vehicle systems, formation control, swarming and flocking, distributed sensor network, attitude alignment of groups of satellites, payload transport, task assignment, air traffic control, and many others $[13,14$, $15,16,17]$

It is noticed in the literature that most consensus protocols focus on the case where all the dynamic agents come to consensus about the value of the so-called consensus variable [1,7]. No matter whether the consensus variable is a vector or not, such consensus protocols are in fact first-order, since the typical consensus protocol adjusts the first derivative of the consensus variable for each agent based on the consensus variable of its neighbors. Despite their widespread importance, there has been little work dealing explicitly with higher-order consensus seeking processes on multiagent networks $[2,12,18,19]$. Under various topological and communication condition settings, these few examples show the convergence conditions that guarantee the second-order or third-order consensus, but provide neither a generic picture nor systematic insight into the connection between the stability of the general high-order consensus and the system parameters such as the network topology and feedback gains.

The motivation for employing high-order integrator to model the intrinsic dynamics of agents in our framework is inspired by the following two reasons. First, from the point of view of system theory, nonlinear systems of a general class (feedback linearizable) can be transformed

\footnotetext{
*Corresponding author e-mail: boboboy@gmail.com
} 
to linear systems via feedback control and transformation of the state vector by using differential-geometric methods [20]. Hence if there exist protocols solving the consensus problems for networks of agents with dynamics described by the high-order integrator, then consensus controllers can be synthesized for nonlinear dynamic networks. Second, observing flocking, schooling, and swarming behavior in nature has truly inspired that biological networks may also need to build consensus on acceleration or even jerk to maintain the collective behaviours in some sudden events (for instance, when one of a fish school is suddenly aware of some source of foods or threats). Therefore it is of both biological and system theoretical interest to pursue the consensus problems for networks of high-order integrator dynamic agents.

In the current paper we investigate arbitrary high-order consensus seeking processes via a frequency domain approach in a systematic manner, which links explicitly the connectivity pattern and control gains to the consensus of multiagent networks. To the best of our knowledge, this is the first work to extend the frequency domain method to arbitrary high-order consensus problems. We then use the derived consensus polynomials to give the general sufficient and necessary stability criterion for the high-order consensus. Moreover, a sufficient condition of desirable time complexity for high-order consensus is given by exploiting the topology properties of underlying networks.

\section{Background and Preliminaries}

Consider $n$ dynamic agents with $k$ th-order dynamics described by

$$
\left\{\begin{array}{l}
\dot{x}_{i}^{(0)}(t)=x_{i}^{(1)}(t), \\
\vdots \\
\dot{x}_{i}^{(k-2)}(t)=x_{i}^{(k-1)}(t), \\
\dot{x}_{i}^{(k-1)}(t)=u_{i}(t),
\end{array}\right.
$$

where $i=1,2, \ldots, n$, and $x_{i}^{(m)}(t) \in R, m=0,1, \ldots, k-1$, and $u_{i}(t) \in R$ denote the information states and the control input of agent $i$, respectively. $x_{i}^{(m)}(t)$ denotes the $m$ th derivative of $x_{i}$ with $x_{i}^{(0)}(t)=x_{i}(t)$. Define the states of the whole network as

$$
\boldsymbol{X}(t)=\left[\left(\boldsymbol{x}^{(0)}(t)\right)^{T}, \ldots,\left(\boldsymbol{x}^{(k-1)}(t)\right)^{T}\right]^{T},
$$

where $\boldsymbol{x}^{(m)}(t)=\left[x_{1}^{(m)}(t), \ldots, x_{n}^{(m)}(t)\right]^{T}, m=0,1, \ldots, k-1$. Then we can decompose $\boldsymbol{x}^{(m)}(t)$ according to the following equation:

$$
\boldsymbol{x}^{(m)}(t)=\bar{x}^{(m)}(t) \mathbf{1}+\boldsymbol{\delta}^{(m)}(t),
$$

where $\bar{x}^{(m)}(t)=\sum_{i} x_{i}^{(m)}(t) / n, \mathbf{1}=[1,1, \ldots, 1]^{T} \in R^{n}$, and $\boldsymbol{\delta}^{(m)}(t) \in R^{n}$. We refer to $e(t)=\sum_{m=0}^{k-1}\left\|\boldsymbol{\delta}^{(m)}(t)\right\|^{2}$ as the overall group disagreement for the $k$ th-order consensus problems, where $\|\cdot\|$ denotes the Euclidean norm. The sets of integers, real numbers, and complex numbers are denoted by $Z, R$, and $C$, respectively.

Information exchange between agents can be naturally modeled by the weighted undirected graph $G=(V, E, \boldsymbol{A})$, where $V=\left\{v_{i}\right\}$ is the set of agents, $\left\{e_{i j}\right\}=E \subseteq V \times V$ is the set of links between the agents and $\boldsymbol{A}$ is the corresponding weighted adjacency matrix. The adjacency matrix $\boldsymbol{A}=\left[a_{i j}\right] \in R^{n \times n}$ is defined such that $a_{i j}>0$ if $\left(v_{j}, v_{i}\right) \in E$, while $a_{i j}=0$ if $\left(v_{j}, v_{i}\right) \notin E$. Therefore the $a_{i j}$ allow our results to be valid and useful for weighted network that is much more general than 0-1 weighted network. Let matrix $\boldsymbol{L}=\left[l_{i j}\right]$ be defined as $l_{i i}=\sum_{j \neq i} a_{i j}$ and $l_{i j}=-a_{i j}$ where $i \neq j$. Following algebraic graph theory, $L$ is positive semi-definite and is called the Laplacian matrix. The set of neighbors of agent $i$ is defined as $N_{i}=\left\{v_{j} \mid\left(v_{j}, v_{i}\right) \in E\right\}$. The degree of the node $v_{i} \in V$ and the average of the degrees of the vertices adjacent to $v_{i}$ are denoted by $d_{i}=\left|N_{i}\right|$ and $m_{i}$, respectively. The graph $G$ does not contain a loop, a link joining an agent to itself.

We propose the following high-order consensus protocol

$$
u_{i}(t)=\sum_{j \in N_{i}} a_{i j} \sum_{m=0}^{k-1} \beta_{m}\left(x_{j}^{(m)}(t)-x_{i}^{(m)}(t)\right),
$$

where $\beta_{m}$ are positive constants denoting the feedback gains of the protocol.

The high-order consensus problem discussed in this paper is defined exactly as follows:

Definition 2.1. ( $k$ th-order consensus). For the network of $k$ th-order integrator systems, consensus is said to be reached globally asymptotically among dynamic agents if $\left|x_{i}^{(m)}(t)-x_{j}^{(m)}(t)\right| \rightarrow 0, m=0,1, \ldots, k-1, \forall i \neq j$ as $t \rightarrow \infty$ for any $\boldsymbol{x}(0)$.

\section{A General Stability Criterion for Arbitrary High-Order Consensus Seeking}

The main result is given in the following theorem.

Theorem 3.1. Consider a network of $n$ dynamic agents with dynamics described by (1). Assume that the network $G=(V, E, \boldsymbol{A})$ is connected and that each agent receives the information from its neighboring agents and applies the control (3). Then the $k$ th-order consensus is achieved, if and only if the following $(n-1)$ polynomials $P_{i}(s)$ are Hurwitz stable for the nonidentically zero eigenvalues $\mu_{i}$ $(i \geq 2)$ of the negative Laplacian matrix $-\boldsymbol{L}$ :

$$
P_{i}(s)=s^{k}-\mu_{i} \sum_{m=0}^{k-1} \beta_{m} s^{m}
$$

Proof. Since the graph $G$ is connected, the Laplacian 
matrix $\boldsymbol{L}$ of $G$ has a simple zero eigenvalue and all the other eigenvalues are positive real numbers. Hence $-\boldsymbol{L}$ has exactly one zero eigenvalue and all the other eigenvalues are negative real numbers. Therefore we write the eigenvalues of $-\boldsymbol{L}$ in the form $\mu_{n} \leq \mu_{n-1} \leq \cdots \leq \mu_{2}<\mu_{1}=0$. Substituting the high-order consensus protocol given by (3) into the dynamics equations of the agents described by (1) yields

$$
\left\{\begin{array}{l}
\dot{\boldsymbol{x}}^{(0)}(t)=\boldsymbol{x}^{(1)}(t) \\
\vdots \\
\dot{\boldsymbol{x}}^{(k-2)}(t)=\boldsymbol{x}^{(k-1)}(t) \\
\dot{\boldsymbol{x}}^{(k-1)}(t)=-\boldsymbol{L} \sum_{m=0}^{k-1} \beta_{m} \boldsymbol{x}^{(m)}(t)
\end{array}\right.
$$

Because of the symmetry of the protocol, we find that $\bar{x}^{(k-1)}$ is an invariant quality. It is noticed that the real-symmetric matrix $-\boldsymbol{L}$ possesses a complete orthonormal set of real eigenvectors. Consequently, we can diagonalize $-\boldsymbol{L}$ with an orthogonal similarity transformation $-\boldsymbol{U}^{T} \boldsymbol{L} \boldsymbol{U}=\boldsymbol{\mu}$ for some orthogonal $\boldsymbol{U}$, where the real-diagonal matrix $\boldsymbol{\mu}=\operatorname{diag}\left\{\mu_{1}, \ldots, \mu_{n}\right\}$. Define $\boldsymbol{y}^{(m)}(t)=\boldsymbol{U}^{T} \boldsymbol{x}^{(m)}(t)$. By employing the above linear transformations, the closed-loop dynamics equations can be decoupled into $n$ noninteracting subsystems

$$
\left\{\begin{array}{l}
\dot{y}_{i}^{(0)}(t)=y_{i}^{(1)}(t) \\
\vdots \\
\dot{y}_{i}^{(k-2)}(t)=y_{i}^{(k-1)}(t) \\
\dot{y}_{i}^{(k-1)}(t)=\mu_{i} \sum_{m=0}^{k-1} \beta_{m} y_{i}^{(m)}(t)
\end{array}\right.
$$

where $i=1,2, \ldots, n$, and $y_{i}^{(m)}(t)$ is the $i$ th component of $\boldsymbol{y}^{(m)}(t)$.

In order to establish the stability of the high-order consensus system, our proof heavily depends on the frequency domain analysis. The approach to the analysis of convergence of the high-order consensus protocol in this paper is to use the Laplace transform. According to (6), we obtain

$$
\begin{aligned}
{\left[\begin{array}{c}
y_{i}^{(0)}(s) \\
\vdots \\
y_{i}^{(k-2)}(s) \\
y_{i}^{(k-1)}(s)
\end{array}\right]-\left[\begin{array}{c}
y_{i}^{(0)}(0) \\
\vdots \\
y_{i}^{(k-2)}(0) \\
y_{i}^{(k-1)}(0)
\end{array}\right]=} & {\left[\begin{array}{cccc}
0 & 1 & \cdots & 0 \\
\vdots & \vdots & \ddots & \vdots \\
0 & 0 & \cdots & 1 \\
\beta_{0} \mu_{i} \cdots & \beta_{k-2} \mu_{i} & \beta_{k-1} \mu_{i}
\end{array}\right] } \\
& {\left[\begin{array}{c}
y_{i}^{(0)}(s) \\
\vdots \\
y_{i}^{(k-2)}(s) \\
y_{i}^{(k-1)}(s)
\end{array}\right] }
\end{aligned}
$$

where $s$ is the Laplace variable. A brief calculation gives

$$
\left[\begin{array}{c}
y_{i}^{(0)}(s) \\
\vdots \\
y_{i}^{(k-2)}(s) \\
y_{i}^{(k-1)}(s)
\end{array}\right]=\left(s \boldsymbol{I}_{n}-\boldsymbol{\Gamma}_{i}(s)\right)^{-1}\left[\begin{array}{c}
y_{i}^{(0)}(0) \\
\vdots \\
y_{i}^{(k-2)}(0) \\
y_{i}^{(k-1)}(0)
\end{array}\right],
$$

where

$$
\Gamma_{i}(s)=\left[\begin{array}{cccc}
0 & 1 & \cdots & 0 \\
\vdots & \vdots & \ddots & \vdots \\
0 & 0 & \cdots & 1 \\
\beta_{0} \mu_{i} & \cdots & \beta_{k-2} \mu_{i} & \beta_{k-1} \mu_{i}
\end{array}\right]
$$

and $\boldsymbol{I}_{n}$ is the $n \times n$ identity matrix. Denote $\boldsymbol{Z}_{i}(s)=s \boldsymbol{I}_{n}-$ $\Gamma_{i}(s)$. Let $\left(s,\left[f_{i 0}, \ldots, f_{i(k-2)}, f_{i(k-1)}\right]^{T}\right)$ be a right MIMO transmission zero of $\boldsymbol{Z}_{i}(s)$ at frequency $s$ in the direction $\left[f_{i 0}, \ldots, f_{i(k-2)}, f_{i(k-1)}\right]^{T} ;$ that is,

$$
\boldsymbol{Z}_{i}(s)\left[f_{i 0}, \ldots, f_{i(k-2)}, f_{i(k-1)}\right]^{T}=\mathbf{0},
$$

where $s \in C$ and $\left[f_{i 0}, \ldots, f_{i(k-2)}, f_{i(k-1)}\right]^{T} \neq \mathbf{0}$. Then we find that

$$
\left\{\begin{array}{l}
s f_{i 0}-f_{i 1}=0 \\
\vdots \\
s f_{i(k-2)}-f_{i(k-1)}=0 \\
-\mu_{i} \sum_{m=0}^{k-1} \beta_{m} f_{i m}+s f_{i(k-1)}=0
\end{array}\right.
$$

and therefore,

$$
\left[s^{k}-\mu_{i} \sum_{m=0}^{k-1} \beta_{m} s^{m}\right] f_{i 0}=0 .
$$

It is obvious that $f_{i 0} \neq 0$. Thus (10) tells us that the poles of the $i$ th subsystem described by (6) can be calculated according to the following important equations

$$
P_{i}(s)=s^{k}-\mu_{i} \sum_{m=0}^{k-1} \beta_{m} s^{m}=0,
$$

where $i=1,2, \ldots, n$.

Following the multivariable control theory, we see that the information states of all the agents achieve the high-order consensus globally asymptotically, if and only if the poles given by (11) (i.e., the poles of the high-order consensus system) are located on the open left half plane (LHP), except for the isolated zero poles associated with $\mu_{1}=0$. And this completes the proof of the theorem.

Remark 3.1. The polynomials $P_{i}(s)=s^{k}-\mu_{i} \sum_{m=0}^{k-1} \beta_{m} s^{m}$ are fundamental to consensus problems and therefore called Consensus Polynomials in the remainder of this paper. Theorem 3.1 clarifies a rigorous connection between the stability of general high-order consensus and 
the system parameters such as the network topology and feedback gains. In addition, it provides us with a general procedure for determining the stability of arbitrary high-order consensus. We construct the consensus polynomials $P_{i}(s)$ and examine their roots. If $\operatorname{Re}(s)<0$ for all the $P_{i}(s)$ with $i \geq 2$, we conclude that the consensus is asymptomatically achievable.

Remark 3.2. Moreover, from Theorem 3.1, we observe that the common assumption used in the literature $[7,12]$ (i.e., $G=(V, E, A)$ is connected and feedback gain constants $\beta_{i}$ are positive) is no longer a sufficient condition for reaching consensus higher than second-order. As the order of the consensus increases, the effects of high-order dynamics of the agents emerge and the conditions for consensus are more and more uncharitable.

\section{Application of the Proposed Stability Criterion}

We stress that Theorem 3.1 completely characterizes the dynamical behavior of arbitrary high-order consensus in a unified framework. Hence it can be used to deduce the algebraic conditions for consensus seeking of any specific order. In order to illustrate this concept, let us consider the fourth-order consensus seeking problem in the following results.

Corollary 4.1. Consider a connected network of fourth-order integrator agents with a topology $G=(V, E, \boldsymbol{A})$. Then the network guided by protocol (3) solves the fourth-order consensus if and only if the following conditions are satisfied:

$$
\mu_{2}<\min \left\{-\beta_{1} /\left(\beta_{2} \beta_{3}\right), \beta_{1}^{2} /\left[\beta_{3}\left(\beta_{0} \beta_{3}-\beta_{1} \beta_{2}\right)\right]\right\},
$$

$$
\beta_{0} \beta_{3}<\beta_{1} \beta_{2} \text {. }
$$

Proof. For the fourth-order consensus (i.e., $k=4$ ), each agent is governed by the fourth-order integrator and driven towards consensus by the distributed protocol

$$
u_{i}(t)=\sum_{j \in N_{i}} a_{i j} \sum_{m=0}^{3} \beta_{m}\left(x_{j}^{(m)}(t)-x_{i}^{(m)}(t)\right) .
$$

According to Theorem 3.1, conditions (12) and (13) are derived from the Routh-Hurwitz criterion for the following consensus polynomials

$$
P_{i}(s)=s^{4}-\mu_{i} \beta_{3} s^{3}-\mu_{i} \beta_{2} s^{2}-\mu_{i} \beta_{1} s-\mu_{i} \beta_{0} .
$$

The proof is complete.

Remark 4.1. For reaching the fourth-order consensus, condition (12) neatly separates questions of consensus protocol from questions of network structure. The structure appears only on the left of the inequality, via the eigenvalues of the graph Laplacian, and the consensus protocol appears only on the right, via feedback gains. Apart from establishing a constraint between the network structure and the consensus protocol, Corollary 4.1 exerts an additional constraint (13) solely on the feedback gains of the protocol.

Remark 4.2. The implication of (13) is somewhat surprising. For instance, if all the feedback gains are set to be a common value, then the consensus will never be achieved no matter how large the feedback gains are. Hence, contrary to common wisdom, increasing the feedback gains is neither sufficient nor necessary to guarantee the high-order consensus.

\section{A Sufficient Condition for High-Order Consensus Using Topology Properties of Networks}

When the underlying network is an unweighed graph, denoted as $G_{u}=\left(V, E, \boldsymbol{A}_{u}\right)$ (i.e., ignoring the weights of the links in the networks), a sufficient condition for the general $k$ th-order consensus is derived as follows. In such a case, the Laplacian matrix $\boldsymbol{L}_{u}\left(G_{u}\right)=\boldsymbol{D}_{u}\left(G_{u}\right)-\boldsymbol{A}_{u}\left(G_{u}\right)$ is the difference of the diagonal matrix of vertex degrees $\boldsymbol{D}_{u}\left(G_{u}\right)$ and the 0-1 adjacency matrix $\boldsymbol{A}_{u}\left(G_{u}\right)$. Furthermore, edge connectivity of the graph $G_{u}$ (i.e., the minimal number of links whose removal would result in losing connectivity of the graphic), is denoted by $e\left(G_{u}\right)$. Now we are ready to present our result on finding the relation between the reachability of the high-order consensus and the topology features of the networks.

Theorem 5.1. Consider a network of finite $n$ dynamic agents with dynamics described by (1). Assume that the network $G_{u}=\left(V, E, \boldsymbol{A}_{u}\right)$ is connected and that each agent receives the information from its neighboring agents and applies the control law (3). If the following four polynomials are Hurwitz stable, then the high-order consensus is achieved:

$$
\begin{aligned}
& K_{1}(s)=l_{0}+l_{1} s+u_{2} s^{2}+u_{3} s^{3}+l_{4} s^{4}+l_{5} s^{5}+\cdots, \\
& K_{2}(s)=u_{0}+u_{1} s+l_{2} s^{2}+l_{3} s^{3}+u_{4} s^{4}+u_{5} s^{5}+\cdots, \\
& K_{3}(s)=l_{0}+u_{1} s+u_{2} s^{2}+l_{3} s^{3}+l_{4} s^{4}+u_{5} s^{5}+\cdots, \\
& K_{4}(s)=u_{0}+l_{1} s+l_{2} s^{2}+u_{3} s^{3}+u_{4} s^{4}+l_{5} s^{5}+\cdots,
\end{aligned}
$$

where

$$
l_{m}=2 e\left(G_{u}\right)(1-\cos (\pi / n)) \beta_{m}
$$

and

$$
u_{m}=\max \left\{d_{i}+m_{i} \mid v_{i} \in V\right\} \beta_{m} .
$$

Proof. Consider the eigenvalues $\mu_{n} \leq \mu_{n-1} \leq \cdots \leq \mu_{2}<$ 
$\mu_{1}=0$ of the $-\boldsymbol{L}_{u}$. According to [21,22], we have

$$
\begin{gathered}
\alpha_{1}=2 e\left(G_{u}\right)(1-\cos (\pi / n)) \leq-\mu_{2}, \\
\alpha_{2}=\max \left\{d_{i}+m_{i} \mid v_{i} \in V\right\} \geq-\mu_{n} .
\end{gathered}
$$

Therefore we find

$$
\alpha_{1} \beta_{m} \leq-\mu_{i} \beta_{m} \leq \alpha_{2} \beta_{m},
$$

where $i=2, \ldots, n$.

Let $l_{m}=\alpha_{1} \beta_{m}$ and $u_{m}=\alpha_{2} \beta_{m}$, and then we see each coefficient of the fundamental consensus polynomials in Theorem 3.1

$$
P_{i}(s)=s^{k}-\mu_{i} \sum_{m=0}^{k-1} \beta_{m} s^{m}=a_{i 0}+\cdots+a_{i(k-1)} s^{k-1}+s^{k}
$$

must take value in the specified interval:

$$
l_{m} \leq a_{i m} \leq u_{m},
$$

for $i=2, \ldots, n$, and $m=0, \ldots, k-1$.

Hence if the four polynomials (i.e., (15) (18)) are Hurwitz stable, Kharitonov's theorem [23] guarantees that the $(n-1)$ polynomials $P_{i}(s)$ are Hurwitz stable for all the nonzero eigenvalues $\mu_{i}(i \geq 2)$ of negative Laplacian matrix $-\boldsymbol{L}_{u}$. Then the direct application of Theorem 3.1 completes the proof.

Remark 5.1. Theorem 5.1 provides a sufficient condition to check the reachability of the high-order consensus but not a necessary one. However, this result has its merits in the following three respects. First, what is somewhat surprising about our result of Theorem 5.1 is that, although in principle we are examining $(n-1)=O(n)$ polynomials for stability (see Theorem 3.1), if the four (independent of the size of the networks) polynomials in Theorem 5.1 are examined to be Hurwitz stable, then we can still arrive at the conclusion. This means the dramatic reduction of time complexity. Second, in practice, edge connectivity and degree sequence of network topology can be estimated or detected efficiently for large-scale real-world networks. For instance, the edge connectivity can be computed via the edge-contraction algorithm [24]. Third, from a point of view of the controller synthesis, it is observed that the feedback gains $\beta_{i}$ are robust to the perturbations of the network topology, as long as the edge connectivity and $\max \left\{d_{i}+m_{i} \mid v_{i} \in V\right\}$ of the network are invariant. Hence, Theorem 5.1 provides a conservative but robust design approach to high-order consensus feedback controllers (i.e., consensus protocols).

\section{Numerical Example and Simulation Results}

We emphasize that the results in this paper characterize the convergence properties for the arbitrary high-order

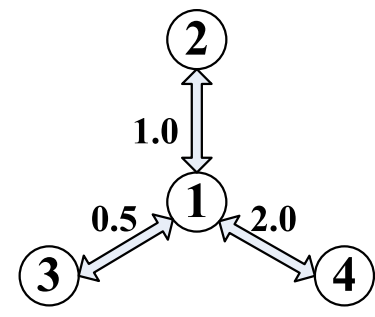

Fig. 1: Undirected communication graph $G$ used for fourth-order consensus problem.

consensus processes on multiagent networks. In order to illustrate these points, we consider solving fourth-order consensus problem of the network of four agents whose communication graph $G$ is shown in Fig. 1. It is easy to see that $G$ is a connected graph. We assume that the numbers on the links are the corresponding weights of the communication links in the graph $G$. Moreover, the agents evolve according to (1) and (3) starting from random initial conditions.

Note that $\mu_{2}=-0.6013$. In Case 1 , we choose $\beta_{0}=1, \beta_{1}=1, \beta_{2}=3$, and $\beta_{3}=4$. Then condition (12) in Corollary 4.1 is satisfied, but it is easily seen that condition (13) is not satisfied. If we choose $\beta_{0}=1$, $\beta_{1}=1, \beta_{2}=2$, and $\beta_{3}=1$ in Case 2, then condition (13) holds but condition (12) does not. Hence the fourth-order consensus can not be achieved in the above two cases. However, if we choose $\beta_{0}=1, \beta_{1}=1, \beta_{2}=3$, and $\beta_{3}=1$ in Case 3 , then all the conditions in Corollary 4.1 are satisfied. As a result, consensus can be reached. Figs. 2, 3, and 4 show the state trajectories $x_{i}^{(m)}$ of the system and the overall group disagreement function $e(t)$ in time for Case 1, 2, and 3, with different feedback gains $\beta_{m}$, $m=0,1,2,3$, values, respectively. Note that although the network topology is connected in all cases, the consensus network is not stable in Case 1 and 2, whereas it is stable in Case 3. Therefore, the feedback gains $\beta_{m}$ must be designed properly to guarantee that the consensus is achieved.

\section{Conclusions}

By a frequency domain method, a rigorous and general convergence analysis was given in this paper for high-order consensus on multiagent networks. We developed sufficient and necessary conditions for the stability of the arbitrary high-order consensus, along with algorithms for checking these conditions. Moreover, a sufficient condition of desirable time complexity for high-order consensus is proposed by exploiting the underlying topology properties. We suggest that insights provided by these results will illuminate the design principles and evolution mechanisms of both natural and engineered dynamic networks, in which consensus is 


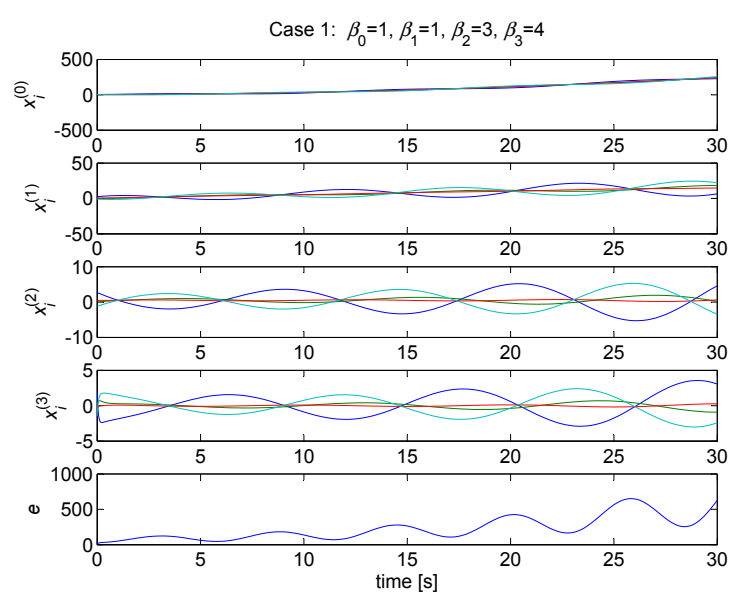

Fig. 2: Evolutions of the fourth-order consensus on graph $G$ given in Fig. 1, with $\beta_{i}$ values (Case 1): $\beta_{0}=1, \beta_{1}=1, \beta_{2}=3$, and $\beta_{3}=4$.

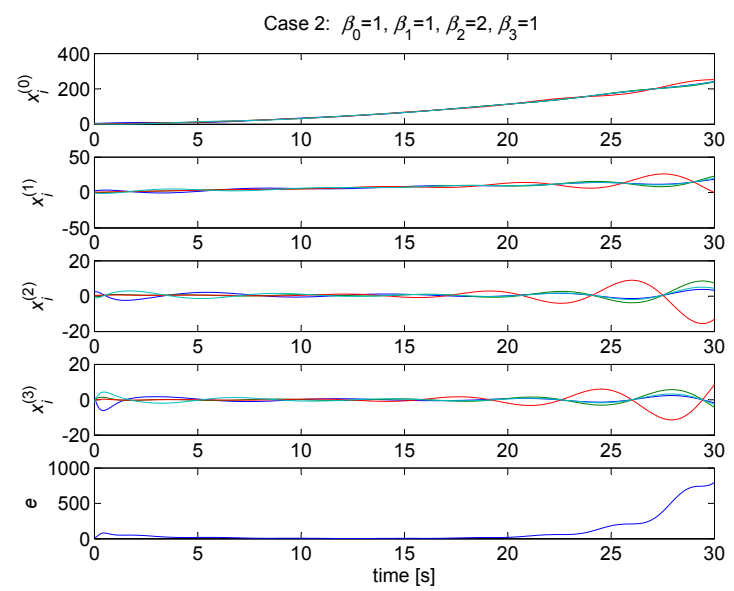

Fig. 3: Evolutions of the fourth-order consensus on graph $G$ given in Fig. 1, with $\beta_{i}$ values (Case 2): $\beta_{0}=1, \beta_{1}=1, \beta_{2}=2$, and $\beta_{3}=1$.

functionally important. In addition, the general high-order consensus problems in dynamical networks with switching topologies will be a topic of future research.

\section{Acknowledgement}

This work was supported by the National Natural Science Foundation of China (Grant No. 61203032), the Natural Science Foundation of Hubei Province (Grant No. 2012FFB05007), and the Fundamental Research Funds for the Central Universities (Grant No. 2010-IV-014).

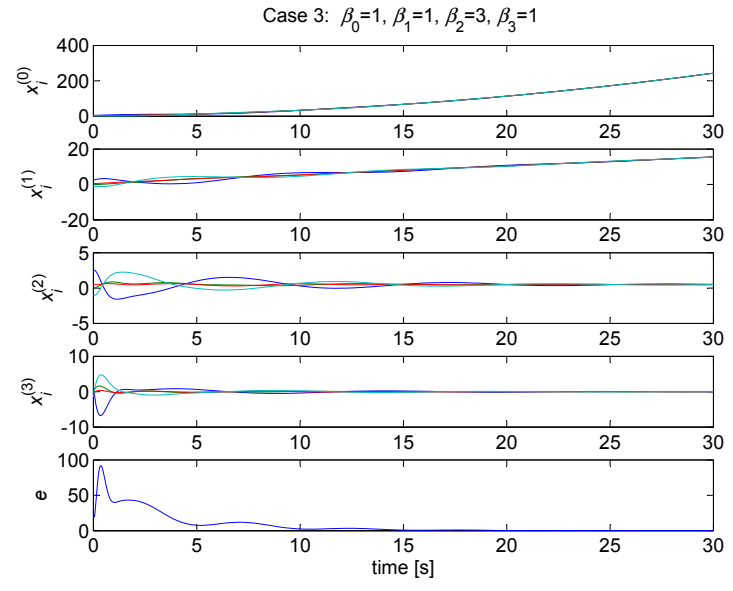

Fig. 4: Evolutions of the fourth-order consensus on graph $G$ given in Fig. 1 , with $\beta_{i}$ values (Case 3): $\beta_{0}=1, \beta_{1}=1, \beta_{2}=3$, and $\beta_{3}=1$.

\section{References}

[1] R. Olfati-Saber and R. M. Murray, Consensus problems in networks of agents with switching topology and time-delays, IEEE Transactions on Automatic Control, 49, 1520-1533 (2004).

[2] W. Ren, Consensus strategies for cooperative control of vehicle formations, IET Control Theory and Applications, 1, 505-512 (2007).

[3] W. Ren and R. W. Beard, Consensus seeking in multiagent systems under dynamically changing interaction topologies, IEEE Transactions on Automatic Control, 50, 655-661 (2005).

[4] W. Ren, R. W. Beard, and E. M. Atkins, Information consensus in multivehicle cooperative control, IEEE Control Systems Magazine, 27, 71-82 (2007).

[5] A. Jadbabaie, J. Lin, and A. S. Morse, Coordination of groups of mobile autonomous agents using nearest neighbor rules, IEEE Transactions on Automatic Control, 48, 988-1001 (2003).

[6] L. Moreau, Stability of multiagent systems with timedependent communication links, IEEE Transactions on Automatic Control, 50, 169-182 (2005).

[7] R. Olfati-Saber, J. A. Fax, and R. M. Murray, Consensus and cooperation in networked multi-agent systems, Proceedings of the IEEE, 95, 215-233 (2007).

[8] D. Bauso, L. Giarre, and R. Pesenti, Non-linear protocols for optimal distributed consensus in networks of dynamic agents, Systems \& Control Letters, 55, 918-928 (2006).

[9] J. H. Seo, H. Shim, and J. Back, Consensus of high-order linear systems using dynamic output feedback compensator: Low gain approach, Automatica, 45, 2659-2664 (2009).

[10] A. V. Savkin, The problem of coordination and consensus achievement in groups of autonomous mobile robots with limited communication, Nonlinear Analysis-Theory Methods \& Applications, 65, 1094-1102 (2006).

[11] M. Porfiri and D. J. Stilwell, Consensus seeking over random weighted directed graphs, IEEE Transactions on Automatic Control, 52, 1767-1773 (2007). 
[12] B. Yang and H. Fang, Forced consensus in networks of double integrator systems with delayed input, Automatica, 46, 629-632 (2010).

[13] H. G. Tanner, A. Jadbabaie, and G. J. Pappas, Flocking in fixed and switching networks, IEEE Transactions on Automatic Control, 52, 863-868 (2007).

[14] H. G. Tanner and D. K. Christodoulakis, Decentralized cooperative control of heterogeneous vehicle groups, Robotics and Autonomous Systems, 55, 811-823 (2007).

[15] V. Gazi and K. M. Passino, Stability analysis of social foraging swarms, IEEE Transactions on Systems Man and Cybernetics Part B-Cybernetics, 34, 539-557 (2004).

[16] V. Gazi and K. M. Passino, Stability analysis of swarms, IEEE Transactions on Automatic Control, 48, 692-697 (2003).

[17] G. Lafferriere, A. Williams, J. Caughman, and J. J. P. Veerman, Decentralized control of vehicle formations, Systems \& Control Letters, 54, 899-910 (2005).

[18] W. Ren, K. L. Moore, and Y. Q. Chen, High-order and model reference consensus algorithms in cooperative control of multivehicle systems, Journal of Dynamic Systems Measurement and Control-Transactions of the ASME, 129, 678-688 (2007).

[19] B. Yang, H. Fang, and H. Wang, Second-order consensus in networks of dynamic agents with communication timedelays, Journal of Systems Engineering and Electronics, 21, 88-94 (2010).

[20] A. Isidori, Nonlinear control systems, Springer Verlag, Berlin, (1995).

[21] M. Fiedler, Algebraic connectivity of graphs, Czechoslovak Mathematical Journal, 23, 298-305 (1973).

[22] R. Merris, A note on Laplacian graph eigenvalues, Linear Algebra and Its Applications, 285, 33-35 (1998).

[23] S. Dasgupta, Kharitonov's theorem revisited, Systems \& Control Letters, 11, 381-384 (1988).

[24] D. R. Karger and C. Stein, A new approach to the minimum cut problem, Journal of the ACM, 43, 601-640 (1996).

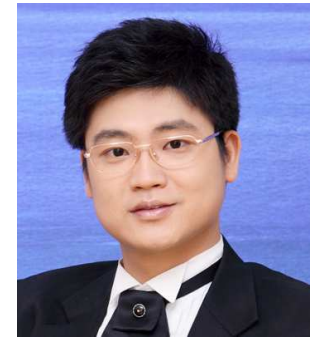

Bo Yang is an Associate Professor at the Wuhan University of Technology, Wuhan, China. He received his B.S. degree in Measuring \& Control Technology and Instrumentations in 2003, and his Ph.D. degree in Control Science and Engineering in 2008, both from the Huazhong University of Science and Technology, Wuhan, China. His research interests include networked systems, nonlinear dynamics and control, complex and intelligent systems, optimization methods, and control applications. $\mathrm{He}$ is a member of IEEE and IEICE. 